\title{
CONVERGENCE IN CAPACITY AND APPLICATIONS
}

\author{
PHAM HOANG HIEP
}

\begin{abstract}
In this article we prove that if $u_{j}, v_{j}, w \in \mathscr{E}(\Omega)$ such that $u_{j}, v_{j} \geq w, \forall j \geq 1$, and $\left|u_{j}-v_{j}\right| \rightarrow 0$ in $C_{n}$-capacity, then $\lim _{j \rightarrow \infty} h\left(\varphi_{1}, \ldots, \varphi_{m}\right)\left[\left(d d^{c} u_{j}\right)^{n}-\left(d d^{c} v_{j}\right)^{n}\right]=0$ in the weak-topology of measures for all $\varphi_{1}, \ldots, \varphi_{m} \in \operatorname{PSH} \cap L_{\mathrm{loc}}^{\infty}(\Omega), h \in C\left(\mathrm{R}^{m}\right)$. We shall then use this result to give some applications.
\end{abstract}

\section{Introduction}

Let $\Omega$ be a bounded hyperconvex domain in $\mathrm{C}^{n}$, by $\operatorname{PSH}(\Omega)$ we denote the set of plurisubharmonic (psh) functions on $\Omega$, and by $\operatorname{PSH}^{-}(\Omega)$ the subclass of negative functions. The complex Monge-Ampère operator $\left(d d^{c}\right)^{n}$ is well defined over the class of locally bounded psh functions, according to the fundamental work of Bedford and Taylor in [3], [4]. In [9], Demailly generalized the work of Bedford and Taylor for the class of locally psh functions with bounded values near the boundary. In [7], Cegrell then introduced a general class $\mathscr{E}(\Omega)$ of psh functions on which the complex Monge-Ampère operator can be defined. The aim of the present paper is to study the convergence within the class $\mathscr{E}(\Omega)$.

In Section 2, we introduce some definitions, and known results that are needed for our paper. Our main result is the following theorem.

Theorem 3.1. Let $u_{j}, v_{j}, w \in \mathscr{E}(\Omega)$ be such that $u_{j}, v_{j} \geq w, \forall j \geq$ 1. Assume that $\left|u_{j}-v_{j}\right| \rightarrow 0$ in $C_{n}$-capacity. Then $\lim _{j \rightarrow \infty} \varphi\left[\left(d d^{c} u_{j}\right)^{n}-\right.$ $\left.\left(d d^{c} v_{j}\right)^{n}\right]=0$ in the weak-topology of measures for all $\varphi \in \operatorname{PSH} \cap L_{\text {loc }}^{\infty}(\Omega)$.

Theorem 3.1 is a generalization of Theorem 1.1 in [8]. As an application we obtain in Theorem 3.3 that if $u_{j}, u, v \in \mathscr{E}(\Omega)$ such that $u_{j} \geq v, \forall j \geq 1$, $\overline{\lim }_{j \rightarrow \infty} u_{j} \leq u$ and $\left(d d^{c} u_{j}\right)^{n} \rightarrow \mu$ in the weak-topology of measures then

$$
\mu \geq 1_{\{u=-\infty\}}\left(d d^{c} u\right)^{n},
$$

in the weak sense of measures.

Received 19 November 2008. 
AcKnOwledgments. The author would like to thank Per Åhag for many fruitful discussions concerning this article. The author is also indebted to the referee for his useful comments that helped to improve the paper.

\section{Preliminaries}

First we recall some elements of pluripotential theory that will be used throughout the paper. All this can be found in [1], [3], [4], [5], [6], [7], [8], [9], [10], [11], [12], [13], [16], [17], [18], [19], and [20].

Notation 2.1. Unless otherwise specified, $\Omega$ will be a bounded hyperconvex domain in $\mathrm{C}^{n}$ meaning that there exists a negative exhaustive psh function for $\Omega$.

Definition 2.2. The $C_{n}$-capacity in the sense of Bedford and Taylor on $\Omega$ is the set function given by

$$
C_{n}(E)=C_{n}(E, \Omega)=\sup \left\{\int_{E}\left(d d^{c} \varphi\right)^{n}: \varphi \in \operatorname{PSH}(\Omega),-1 \leq \varphi \leq 0\right\}
$$

for every Borel set $E$ in $\Omega$. It is known [4] that

$$
C_{n}(E)=\int_{\Omega}\left(d d^{c} h_{E, \Omega}^{*}\right)^{n} .
$$

where $h_{E, \Omega}^{*}$ is the relative extremal psh function for $E$ (relative to $\Omega$ ) defined as the smallest upper semicontinuous majorant of $h_{E, \Omega}$

$$
h_{E, \Omega}(z)=\sup \left\{\varphi(z): \varphi \in \operatorname{PSH}^{-}(\Omega), \varphi \leq-1 \text { on } E\right\} .
$$

The following definition was introduced in [19]: A sequence $u_{j} \in \mathrm{PSH}^{-}(\Omega)$ converges to $u$ in $C_{n}$-capacity if

$$
C_{n}\left(K \cap\left\{\left|u_{j}-u\right|>\delta\right\}\right) \rightarrow 0 \text { as } j \rightarrow+\infty, \forall K \subset \subset \Omega, \forall \delta>0 .
$$

A family of positive measures $\left\{\mu_{\alpha}\right\}$ on $\Omega$ is said to be uniformly absolutely continuous with respect to $C_{n}$-capacity in a set $E \subset \Omega$ if for every $\epsilon>0$ there exists $\delta>0$ such that for each Borel subset $F \subset E$ with $C_{n}(F)<\delta$ the inequality $\mu_{\alpha}(F)<\epsilon$ holds for all $\alpha$. We write $\mu_{\alpha} \ll C_{n}$ in $E$ uniformly for $\alpha$.

Definition 2.3. The following classes of psh functions were introduced by Cegrell in [5], [6] and [7]:

$$
\mathscr{E}_{0}=\mathscr{E}_{0}(\Omega)=\left\{\varphi \in \operatorname{PSH}^{-}(\Omega) \cap L^{\infty}(\Omega): \lim _{z \rightarrow \partial \Omega} \varphi(z)=0, \int_{\Omega}\left(d d^{c} \varphi\right)^{n}<+\infty\right\},
$$




$$
\begin{gathered}
\mathscr{F}=\mathscr{F}(\Omega)=\left\{\varphi \in \operatorname{PSH}^{-}(\Omega): \exists \mathscr{E}_{0}(\Omega) \ni \varphi_{j} \backslash \varphi, \sup _{j \geq 1} \int_{\Omega}\left(d d^{c} \varphi_{j}\right)^{n}<+\infty\right\}, \\
\mathscr{E}=\mathscr{E}(\Omega)=\left\{\varphi \in \operatorname{PSH}^{-}(\Omega): \exists \varphi_{K} \in \mathscr{F}(\Omega)\right. \\
\text { such that } \left.\varphi_{K}=\varphi \text { on } K, \forall K \subset \subset \Omega\right\}, \\
\delta \operatorname{PSH} \cap L^{\infty}(\Omega)=\left\{\varphi-\psi: \varphi, \psi \in \operatorname{PSH} \cap L^{\infty}(\Omega)\right\}, \\
\delta \mathscr{E}_{0}(\Omega)=\left\{\varphi-\psi: \varphi, \psi \in \mathscr{E}_{0}(\Omega)\right\} .
\end{gathered}
$$

Next we introduce some results needed for our work.

Proposition 2.4. i) Let $\varphi, \psi \in \delta \operatorname{PSH} \cap L^{\infty}(\Omega)$. Then $\varphi \psi \in \delta \operatorname{PSH} \cap$ $L^{\infty}(\Omega)$.

ii) Let $\varphi, \psi \in \delta \mathscr{E}_{0}(\Omega)$. Then $\varphi \psi \in \delta \mathscr{E}_{0}(\Omega)$.

Proof. i) Without loss of generality we can assume that $\varphi, \psi \in \mathrm{PSH} \cap$ $L^{\infty}(\Omega)$. Set $c=\sup \{|\varphi(z)|+|\psi(z)|: z \in \Omega\}$. We have

$$
\varphi \psi=\frac{1}{2}\left[(\varphi+\psi+c)^{2}-(\varphi+c)^{2}-(\psi+c)^{2}+c^{2}\right] .
$$

Hence $\varphi \psi \in \delta \operatorname{PSH} \cap L^{\infty}(\Omega)$.

ii) Without loss of generality we can assume that $\varphi, \psi \in \delta \mathscr{E}_{0}(\Omega)$. Set $c=\sup \{|\varphi(z)|+|\psi(z)|: \quad z \in \Omega\}$. We prove that $(\varphi+c)^{2}-c^{2} \in \mathscr{E}_{0}(\Omega)$. Indeed, we have $d d^{c}\left[(\varphi+c)^{2}-c^{2}\right]=2\left[(\varphi+c) d d^{c} \varphi+d \varphi \wedge d^{c} \varphi\right] \geq 0$. Thus

$$
\begin{aligned}
\int_{\Omega}\left(d d^{c}\right. & {\left.\left[(\varphi+c)^{2}-c^{2}\right]\right)^{n} } \\
& =\int_{\Omega} 2^{n}\left[(\varphi+c)^{n}\left(d d^{c} \varphi\right)^{n}+n(\varphi+c)^{n-1} d \varphi \wedge d^{c} \varphi \wedge\left(d d^{c} \varphi\right)^{n-1}\right] \\
& \leq \int_{\Omega} 2^{n} c^{n}\left(d d^{c} \varphi\right)^{n}+2^{n} n c^{n-1} \int_{\Omega} d \varphi \wedge d^{c} \varphi \wedge\left(d d^{c} \varphi\right)^{n-1} \\
& =\int_{\Omega} 2^{n} c^{n}\left(d d^{c} \varphi\right)^{n}+2^{n} n c^{n-1} \int_{\Omega}-\varphi\left(d d^{c} \varphi\right)^{n} \\
& \leq \int_{\Omega} 2^{n} c^{n}(n+1)\left(d d^{c} \varphi\right)^{n}<+\infty .
\end{aligned}
$$

Moreover, since $\lim _{z \rightarrow \partial \Omega}\left[(\varphi+c)^{2}-c^{2}\right]=0$ we get $(\varphi+c)^{2}-c^{2} \in \mathscr{E}_{0}(\Omega)$. On the other hand, we have

$$
\varphi \psi=\frac{(\varphi+\psi+c)^{2}-c^{2}}{2}-\frac{(\varphi+c)^{2}-c^{2}}{2}-\frac{(\psi+c)^{2}-c^{2}}{2},
$$

Hence $\varphi \psi \in \delta \mathscr{E}_{0}(\Omega)$. 
Proposition 2.5. Let $u_{1}, \ldots, u_{n} \in \mathscr{F}(\Omega)$ be such that $u_{1}, \ldots, u_{k} \geq-1$. Then

$$
\int_{B} d d^{c} u_{1} \wedge \cdots \wedge d d^{c} u_{n} \leq\left[\int_{\Omega}\left(d d^{c} u_{k+1}\right)^{n}\right]^{\frac{1}{n}} \cdots\left[\int_{\Omega}\left(d d^{c} u_{n}\right)^{n}\right]^{\frac{1}{n}} C_{n}(B)^{\frac{k}{n}},
$$

for all Borel sets $B \subset \Omega$.

Proof. For each $u \in \mathscr{E}(\Omega)$ we set

$$
h_{B, u}(z)=\sup \left\{\varphi(z): \varphi \in \operatorname{PSH}^{-}(\Omega), \varphi \leq u \text { on } B\right\} .
$$

For each open set $B \subset \subset \Omega$, by Corollary 5.6 in [7] we get

$$
\begin{aligned}
\int_{B} d d^{c} & u_{1} \wedge \cdots \wedge d d^{c} u_{n} \\
& =\int_{B} d d^{c} h_{B, u_{1}} \wedge \cdots \wedge d d^{c} h_{B, u_{k}} \wedge d d^{c} u_{k+1} \wedge \cdots d d^{c} u_{n} \\
& \leq \int_{\Omega} d d^{c} h_{B, u_{1}} \wedge \cdots \wedge d d^{c} h_{B, u_{k}} \wedge d d^{c} u_{k+1} \wedge \cdots \wedge d d^{c} u_{n} \\
& \leq \int_{\Omega}\left(d d^{c} h_{B}\right)^{k} \wedge d d^{c} u_{k+1} \wedge \cdots \wedge d d^{c} u_{n} \\
& \leq\left[\int_{\Omega}\left(d d^{c} h_{B}\right)^{n}\right]^{\frac{k}{n}}\left[\int_{\Omega}\left(d d^{c} u_{k+1}\right)^{n}\right]^{\frac{1}{n}} \cdots\left[\int_{\Omega}\left(d d^{c} u_{n}\right)^{n}\right]^{\frac{1}{n}} \\
& \leq\left[\int_{\Omega}\left(d d^{c} u_{k+1}\right)^{n}\right]^{\frac{1}{n}} \cdots\left[\int_{\Omega}\left(d d^{c} u_{n}\right)^{n}\right]^{\frac{1}{n}}\left[C_{n}(B)\right]^{\frac{k}{n}} .
\end{aligned}
$$

Hence

$$
\int_{B} d d^{c} u_{1} \wedge \cdots \wedge d d^{c} u_{n} \leq\left[\int_{\Omega}\left(d d^{c} u_{k+1}\right)^{n}\right]^{\frac{1}{n}} \cdots\left[\int_{\Omega}\left(d d^{c} u_{n}\right)^{n}\right]^{\frac{1}{n}}\left[C_{n}(B)\right]^{\frac{k}{n}} .
$$

for all Borel sets $B \subset \Omega$.

Proposition 2.6. Let $u, v, w_{1}, \ldots, w_{n-1} \in \mathscr{F}(\Omega), s>0$ be such that $u \geq v$. Then

$$
\int_{\Omega}[\max (v,-s)-v] d d^{c} u \wedge T \leq \int_{\Omega}[\max (2 v,-s)-2 v] d d^{c} v \wedge T,
$$

where $T=d d^{c} w_{1} \wedge \cdots \wedge d d^{c} w_{n-1}$. 
Proof. By Stokes formula and Theorem 4.1 in [15] we get

$$
\begin{aligned}
\int_{\Omega}[\max (v,-s)-v] d d^{c} u \wedge T & =\int_{\Omega}-u d d^{c}[v-\max (v,-s)] \wedge T \\
& =\int_{\{v \leq-s\}}-u d d^{c}[v-\max (v,-s)] \wedge T \\
& \leq \int_{\{v \leq-s\}}-u d d^{c} v \wedge T \\
& \leq \int_{\{v \leq-s\}}-v d d^{c} v \wedge T \\
& \leq \int_{\{v \leq-s\}}[\max (2 v,-s)-2 v] d d^{c} v \wedge T \\
& \leq \int_{\Omega}[\max (2 v,-s)-2 v] d d^{c} v \wedge T .
\end{aligned}
$$

We generalize Theorem 3.1 in [13]:

Proposition 2.7. Let $\mathscr{E}(\Omega) \ni u_{j} \rightarrow u \in \mathrm{PSH}^{-}(\Omega)$ in $C_{n}$-capacity. Assume that $\left(d d^{c} u_{j}\right)^{n} \rightarrow \mu$ in the weak-topology of measures. Then

$$
\mu \geq 1_{\{u>v\}}\left(d d^{c} \max (u, v)\right)^{n},
$$

in the weak sense of measures for all $v \in \mathrm{PSH}^{-} \cap L^{\infty}(\Omega)$, where $1_{E}$ is the characteristic function for the set $E$.

Proof. By Theorem 4.1 in [15] we get

$$
\begin{aligned}
\left(d d^{c} u_{j}\right)^{n} \geq & 1_{\left\{u_{j}>v-1\right\}}\left(d d^{c} u_{j}\right)^{n} \\
= & 1_{\left\{u_{j}>v-1\right\}}\left(d d^{c} \max \left(u_{j}, v-1\right)\right)^{n} \\
= & \left(d d^{c} \max \left(u_{j}, v-1\right)\right)^{n}-1_{\left\{u_{j} \leq v-1\right\}}\left(d d^{c} \max \left(u_{j}, v-1\right)\right)^{n} \\
\geq & \left(d d^{c} \max \left(u_{j}, v-1\right)\right)^{n}-1_{\left\{u_{j} \leq v-1\right\} \cap\left\{\left|u_{j}-u\right| \leq 1\right\}}\left(d d^{c} \max \left(u_{j}, v-1\right)\right)^{n} \\
& \quad-1_{\left\{\left|u_{j}-u\right|>1\right\}}\left(d d^{c} \max \left(u_{j}, v-1\right)\right)^{n} \\
\geq & \left(d d^{c} \max \left(u_{j}, v-1\right)\right)^{n}-1_{\{u \leq v\}}\left(d d^{c} \max \left(u_{j}, v-1\right)\right)^{n} \\
& \quad-1_{\left\{\left|u_{j}-u\right|>1\right\}}\left(d d^{c} \max \left(u_{j}, v-1\right)\right)^{n} .
\end{aligned}
$$

From the quasicontinuity of $u$ and $v$ (Theorem 3.5 in [4]) and from $\left(d d^{c} \max \left(u_{j}\right.\right.$, $v-1))^{n} \ll C_{n}$ in $\Omega$ uniformly for $j$ we get

$$
\varlimsup_{j \rightarrow \infty} 1_{\{u \leq v\}}\left(d d^{c} \max \left(u_{j}, v-1\right)\right)^{n} \leq 1_{\{u \leq v\}}\left(d d^{c} \max (u, v-1)\right)^{n} .
$$


On the other hand, since $u_{j} \rightarrow u$ in $C_{n}$-capacity and $\left(d d^{c} \max \left(u_{j}, v-1\right)\right)^{n} \ll$ $C_{n}$ in $\Omega$ uniformly for $j$ we get

$$
\lim _{j \rightarrow \infty} 1_{\left\{\left|u_{j}-u\right|>1\right\}}\left(d d^{c} \max \left(u_{j}, v-1\right)\right)^{n}=0 .
$$

Moreover, by Theorem 1.1 in [8] and Theorem 4.1 in [15] we obtain

$$
\begin{aligned}
\mu & \geq\left(d d^{c} \max (u, v-1)\right)^{n}-1_{\{u \leq v\}}\left(d d^{c} \max (u, v-1)\right)^{n} \\
& =1_{\{u>v\}}\left(d d^{c} \max (u, v-1)\right)^{n} \\
& =1_{\{u>v\}}\left(d d^{c} \max (u, v)\right)^{n} .
\end{aligned}
$$

Let $X$ be a compact Kähler manifold with a fundamental form $\omega=\omega_{X}$ such that $\int_{X} \omega^{n}=1$. An upper semicontinuous function $\varphi: X \rightarrow[-\infty,+\infty)$ is called $\omega$-plurisubharmonic ( $\omega$-psh) if $\varphi \in L^{1}(X)$ and $\omega+d d^{c} \varphi \geq 0$. In [18] Kołodziej introduced the capacity $C_{X, \omega}$ on $X$ by

$$
C_{X}(E)=C_{X, \omega}(E)=\sup \left\{\int_{E} \omega_{\varphi}^{n}: \varphi \in \operatorname{PSH}(X, \omega),-1 \leq \varphi \leq 0\right\},
$$

where $\omega_{\varphi}^{n}=\left(\omega+d d^{c} \varphi\right)^{n}$ and $n=\operatorname{dim} X$. In [10] Guedj and Zeriahi proved that $C_{X}$ is a Choquet capacity on $X$ and

$$
C_{X}(E)=\int_{X}\left(-h_{E, \omega}^{*}\right) \omega_{h_{E, \omega}^{*}}^{n} .
$$

where $h_{E, \omega}^{*}$ denotes the upper semicontinuous regularization of $h_{E, \omega}$ given by

$$
h_{E, \omega}(z)=\sup \left\{\varphi(z): \varphi \in \mathrm{PSH}^{-}(X, \omega),\left.\varphi\right|_{E} \leq-1\right\} .
$$

In [11] they introduced the new class of $\omega$-psh functions

$$
\mathscr{E}(X, \omega)=\left\{\varphi \in \operatorname{PSH}(X, \omega): \lim _{j \rightarrow \infty} \int_{\{\varphi>-j\}} \omega_{\max (\varphi,-j)}^{n}=1\right\} .
$$

They proved that the complex Monge-Ampère is well defined on $\mathscr{E}(X, \omega)$ by

$$
\omega_{\varphi}^{n}=\lim _{j \rightarrow \infty} 1_{\{\varphi>-j\}} \omega_{\max (\varphi,-j)}^{n} .
$$

From the proof of Proposition 2.7 we give a simple proof of Theorem 1.9 in [11] (see [20]):

Proposition 2.8. Let $\mathscr{E}(X, \omega) \ni u_{j} \rightarrow u \in \mathscr{E}(X, \omega)$ in $C_{X}$-capacity. Then $\omega_{u_{j}}^{n} \rightarrow \omega_{u}^{n}$ in the weak-topology of measures. 
Proof. Without loss of generality we can assume that $\omega_{u_{j}}^{n} \rightarrow \mu$ in the weak-topology of measures. We have

$$
\begin{aligned}
\omega_{u_{j}}^{n} \geq & 1_{\left\{u_{j}>-k-1\right\}} \omega_{\max \left(u_{j},-k-1\right)}^{n} \\
= & \omega_{\max \left(u_{j},-k-1\right)}^{n}-1_{\left\{u_{j} \leq-k-1\right\}} \omega_{\max \left(u_{j},-k-1\right)}^{n} \\
\geq & \omega_{\max \left(u_{j},-k-1\right)}^{n}-1_{\left\{u_{j} \leq-k-1\right\} \cap\left\{\left|u_{j}-u\right| \leq 1\right\}} \omega_{\max \left(u_{j},-k-1\right)}^{n} \\
& \quad-1_{\left\{\left|u_{j}-u\right|>1\right\}} \omega_{\max \left(u_{j},-k-1\right)}^{n} \\
& \geq \omega_{\max \left(u_{j},-k-1\right)}^{n}-1_{\{u \leq-k\}} \omega_{\max \left(u_{j},-k-1\right)}^{n}-1_{\left\{\left|u_{j}-u\right|>1\right\}} \omega_{\max \left(u_{j},-k-1\right)}^{n} .
\end{aligned}
$$

From the quasicontinuity of $u$ (Corollary 3.8 in [10]) and from $\omega_{\max \left(u_{j},-k-1\right)}^{n} \ll$ $C_{n}$ in $\Omega$ uniformly for $j$ we get

$$
\varlimsup_{j \rightarrow \infty} 1_{\{u \leq-k\}} \omega_{\max \left(u_{j},-k-1\right)}^{n} \leq 1_{\{u \leq-k\}} \omega_{\max (u,-k-1)}^{n} .
$$

On the other hand, since $u_{j} \rightarrow u$ in $C_{n}$-capacity and $\omega_{\max \left(u_{j},-k-1\right)}^{n} \ll C_{n}$ in $\Omega$ uniformly for $j$ we get

$$
\lim _{j \rightarrow \infty} 1_{\left\{\left|u_{j}-u\right|>1\right\}} \omega_{\max \left(u_{j},-k-1\right)}^{n}=0 .
$$

Moreover, by Theorem 1.1 in [8] we obtain

$$
\begin{aligned}
\mu \geq \omega_{\max (u,-k-1)}^{n}-1_{\{u \leq-k\}} \omega_{\max (u,-k-1)}^{n} & =1_{\{u>-k\}} \omega_{\max (u,-k-1)}^{n} \\
& =1_{\{u>-k\}} \omega_{\max (u,-k)}^{n},
\end{aligned}
$$

Letting $k \rightarrow \infty$ we obtain $\mu \geq \omega_{u}^{n}$. Moreover, we have $\mu(X)=\omega_{u}^{n}(X)=$ 1. Hence $\mu=\omega_{u}^{n}$.

\section{Convergence in capacity}

We start with the first result which is a generalization of Theorem 1.1 in [8].

Theorem 3.1. Let $u_{j}, v_{j}, w \in \mathscr{E}(\Omega)$ be such that $u_{j}, v_{j} \geq w, \forall j \geq$ 1. Assume that $\left|u_{j}-v_{j}\right| \rightarrow 0$ in $C_{n}$-capacity. Then $\lim _{j \rightarrow \infty} \varphi\left[\left(d d^{c} u_{j}\right)^{n}-\right.$ $\left.\left(d d^{c} v_{j}\right)^{n}\right]=0$ in the weak-topology of measures for all $\varphi \in \operatorname{PSH} \cap L_{\mathrm{loc}}^{\infty}(\Omega)$.

Proof. As in the proof of Proposition 2.5 in [13] we can assume that $w \in$ $\mathscr{F}(\Omega)$ and $\varphi \in \mathscr{E}_{0}(\Omega)$. We prove that

$$
\lim _{j \rightarrow \infty} \int_{\Omega} \varphi\left[\left(d d^{c} u_{j}\right)^{n}-\left(d d^{c} v_{j}\right)^{n}\right]=0,
$$


for all $\varphi \in \mathscr{E}_{0}(\Omega)$. We can assume that $\varphi \geq-1$. For each $s>0$ we have

$$
\int_{\Omega} \varphi\left[\left(d d^{c} u_{j}\right)^{n}-\left(d d^{c} v_{j}\right)^{n}\right]=A_{j s}+B_{j s}+C_{j s}
$$

where

$$
\begin{aligned}
A_{j s} & =\int_{\Omega} \varphi\left[\left(d d^{c} u_{j}\right)^{n}-\left(d d^{c} \max \left(u_{j},-s\right)\right)^{n}\right], \\
B_{j s} & =\int_{\Omega} \varphi\left[\left(d d^{c} \max \left(v_{j},-s\right)\right)^{n}-\left(d d^{c} v_{j}\right)^{n}\right], \\
C_{j s} & =\int_{\Omega} \varphi\left[\left(d d^{c} \max \left(u_{j},-s\right)\right)^{n}-\left(d d^{c} \max \left(v_{j},-s\right)\right)^{n}\right] .
\end{aligned}
$$

By Stokes formula and Proposition 2.6 we get

$$
\begin{aligned}
\left|A_{j s}\right| & \mid \int_{\Omega} \varphi\left[d d^{c} u_{j}-d d^{c} \max \left(u_{j},-s\right)\right] \\
& \wedge\left[\sum_{k=0}^{n-1}\left(d d^{c} u_{j}\right)^{k} \wedge\left(d d^{c} \max \left(u_{j},-s\right)\right)^{n-1-k}\right] \mid \\
& =\int_{\Omega}\left[\max \left(u_{j},-s\right)-u_{j}\right] d d^{c} \varphi \\
& \wedge\left[\sum_{k=0}^{n-1}\left(d d^{c} u_{j}\right)^{k} \wedge\left(d d^{c} \max \left(u_{j},-s\right)\right)^{n-1-k}\right] \\
\leq & \sum_{k=0}^{n-1} \int_{\Omega}[\max (w,-s)-w] d d^{c} \varphi \wedge\left(d d^{c} u_{j}\right)^{k} \wedge\left(d d^{c} \max \left(u_{j},-s\right)\right)^{n-1-k} \\
\leq & \sum_{k=0}^{n-1} \int_{\Omega}[\max (2 w,-s)-2 w] d d^{c} \varphi \wedge d d^{c} w \\
& \wedge\left(d d^{c} u_{j}\right)^{k-1} \wedge\left(d d^{c} \max \left(u_{j},-s\right)\right)^{n-1-k} \\
\leq & \ldots \int_{\Omega}\left[\max \left(2^{n-1} w,-s\right)-2^{n-1} w\right] d d^{c} \varphi \wedge\left(d d^{c} w\right)^{n-1} \\
= & n \int_{\Omega} \varphi d d^{c}\left[\max \left(2^{n-1} w,-s\right)-2^{n-1} w\right] \wedge\left(d d^{c} w\right)^{n-1} .
\end{aligned}
$$


Similarly we have

$$
\left|B_{j s}\right| \leq n \int_{\Omega} \varphi d d^{c}\left[\max \left(2^{n-1} w,-s\right)-2^{n-1} w\right] \wedge\left(d d^{c} w\right)^{n-1} .
$$

To simplify the notation we set

$$
\begin{aligned}
\varphi_{\epsilon} & =\max (\varphi,-\epsilon) \\
T_{j s} & =\sum_{k=0}^{n-1}\left(d d^{c} \max \left(u_{j},-s\right)\right)^{k} \wedge\left(d d^{c} \max \left(v_{j},-s\right)\right)^{n-1-k}
\end{aligned}
$$

Next by Stokes formula and Proposition 2.5 we get

$$
\begin{aligned}
& \left|C_{j s}\right| \\
& =\left|\int_{\Omega}\left[\max \left(u_{j},-s\right)-\max \left(v_{j},-s\right)\right] \wedge d d^{c} \varphi \wedge T_{j s}\right| \\
& \leq \int_{\Omega}\left|\max \left(u_{j},-s\right)-\max \left(v_{j},-s\right)\right| d d^{c} \varphi \wedge T_{j s} \\
& \leq \int_{\Omega} \epsilon d d^{c} \varphi \wedge T_{j s}+\int_{\left\{\left|u_{j}-v_{j}\right|>\epsilon\right\}}\left|\max \left(u_{j},-s\right)-\max \left(v_{j},-s\right)\right| d d^{c} \varphi \wedge T_{j s} \\
& \leq n \epsilon \int_{\Omega} d d^{c} \varphi \wedge\left(d d^{c} w\right)^{n-1} \\
& +\int_{\left\{\left|u_{j}-v_{j}\right|>\epsilon\right\}}\left|\max \left(u_{j},-s\right)-\max \left(v_{j},-s\right)\right|\left[d d^{c} \varphi-d d^{c} \varphi_{\epsilon}\right] \wedge T_{j s} \\
& +\int_{\left\{\left|u_{j}-v_{j}\right|>\epsilon\right\}}\left|\max \left(u_{j},-s\right)-\max \left(v_{j},-s\right)\right| d d^{c} \varphi_{\epsilon} \wedge T_{j s} \\
& \leq n \epsilon \int_{\Omega} d d^{c} \varphi \wedge\left(d d^{c} w\right)^{n-1}+\int_{\left\{\left|u_{j}-v_{j}\right|>\epsilon\right\} \cap\{\varphi \leq-\epsilon\}} 2 s\left[d d^{c} \varphi+d d^{c} \varphi_{\epsilon}\right] \wedge T_{j s} \\
& +\int_{\Omega}-2 w d d^{c} \varphi_{\epsilon} \wedge T_{j s} \\
& \leq n \epsilon \int_{\Omega} d d^{c} \varphi \wedge\left(d d^{c} w\right)^{n-1} \\
& +4 \operatorname{sn}\left[\int_{\Omega}\left(d d^{c} w\right)^{n}\right]^{\frac{n-1}{n}}\left[C_{n}\left(\left\{\left|u_{j}-v_{j}\right|>\epsilon\right\} \cap\{\varphi \leq-\epsilon\}\right)\right]^{\frac{1}{n}} \\
& +2 n \int_{\Omega}-w d d^{c} \varphi_{\epsilon} \wedge\left(d d^{c} w\right)^{n-1}
\end{aligned}
$$




$$
\begin{aligned}
= & n \epsilon \int_{\Omega} d d^{c} \varphi \wedge\left(d d^{c} w\right)^{n-1} \\
& +4 s n\left[\int_{\Omega}\left(d d^{c} w\right)^{n}\right]^{\frac{n-1}{n}}\left[C_{n}\left(\left\{\left|u_{j}-v_{j}\right|>\epsilon\right\} \cap\{\varphi \leq-\epsilon\}\right)\right]^{\frac{1}{n}} \\
& +2 n \int_{\Omega}-\varphi_{\epsilon}\left(d d^{c} w\right)^{n} \\
\leq & n \epsilon \int_{\Omega} d d^{c} \varphi \wedge\left(d d^{c} w\right)^{n-1} \\
& +4 s n\left[\int_{\Omega}\left(d d^{c} w\right)^{n}\right]^{\frac{n-1}{n}}\left[C_{n}\left(\left\{\left|u_{j}-v_{j}\right|>\epsilon\right\} \cap\{\varphi \leq-\epsilon\}\right)\right]^{\frac{1}{n}} \\
& +2 n \epsilon \int_{\Omega}\left(d d^{c} w\right)^{n} .
\end{aligned}
$$

Hence

$$
\varlimsup_{j \rightarrow \infty} C_{j s} \leq n \epsilon \int_{\Omega} d d^{c} \varphi \wedge\left(d d^{c} w\right)^{n-1}+2 n \epsilon \int_{\Omega}\left(d d^{c} w\right)^{n}
$$

for all $\epsilon>0$. Letting $\epsilon \rightarrow 0$ we have

$$
\lim _{j \rightarrow \infty} C_{j s}=0 .
$$

Combining these inequalities we obtain

$$
\begin{aligned}
& \varlimsup_{j \rightarrow \infty}\left|\int_{\Omega} \varphi\left[\left(d d^{c} u_{j}\right)^{n}-\left(d d^{c} v_{j}\right)^{n}\right]\right| \\
& \leq 2 n \int_{\Omega} \varphi d d^{c}\left[\max \left(2^{n-1} w,-s\right)-2^{n-1} w\right] \wedge\left(d d^{c} w\right)^{n-1},
\end{aligned}
$$

for all $s>0$. Letting $s \rightarrow \infty$ by Proposition 5.1 in [7] we have

$$
\lim _{j \rightarrow \infty} \int_{\Omega} \varphi\left[\left(d d^{c} u_{j}\right)^{n}-\left(d d^{c} v_{j}\right)^{n}\right]=0 .
$$

Moreover, from $C_{0}^{\infty}(\Omega) \subset \delta \mathscr{E}_{0}(\Omega)$ and from Proposition 2.4 ii) we obtain

$$
\lim _{j \rightarrow \infty} \int_{\Omega} f \varphi\left[\left(d d^{c} u_{j}\right)^{n}-\left(d d^{c} v_{j}\right)^{n}\right]=0,
$$

for all $f \in C_{0}^{\infty}(\Omega)$.

Corollary 3.2. Let $u_{j}, v_{j}, w \in \mathscr{E}(\Omega)$ be such that $u_{j}, v_{j} \geq w, \forall j \geq 1$. Assume that $\left|u_{j}-v_{j}\right| \rightarrow 0$ in $C_{n}$-capacity. Then $\lim _{j \rightarrow \infty} h\left(\varphi_{1}, \ldots, \varphi_{m}\right)\left[\left(d d^{c} u_{j}\right)^{n}\right.$ 
- $\left.\left(d d^{c} v_{j}\right)^{n}\right]=0$ in the weak-topology of measures for all $\varphi_{1}, \ldots, \varphi_{m} \in$ $\operatorname{PSH} \cap L_{l o c}^{\infty}(\Omega), h \in C\left(\mathrm{R}^{m}\right)$.

Proof. As in the proof of Proposition 2.5 in [13] we can assume that $w \in \mathscr{F}(\Omega)$ and $\varphi_{1}, \ldots, \varphi_{m} \in \operatorname{PSH} \cap L^{\infty}(\Omega)$. Set $A=\sup \left\{\max \left(\left|\varphi_{1}(z)\right|, \ldots\right.\right.$, $\left.\left.\left|\varphi_{m}(z)\right|\right): z \in \Omega\right\}$. We choose a sequence of polynomials $P_{j}$ such that

$$
\lim _{j \rightarrow \infty} \sup \left\{\left|P_{j}(x)-h(x)\right|: x \in[-A, A]^{m}\right\}=0 .
$$

On the other hand, by Proposition 2.4 i) we have $P_{j}\left(\varphi_{1}, \ldots, \varphi_{m}\right) \in \delta$ PSH $\cap$ $L^{\infty}(\Omega)$. Moreover, by Theorem 3.1 we obtain $\lim _{j \rightarrow \infty} h\left(\varphi_{1}, \ldots, \varphi_{m}\right)$ $\left[\left(d d^{c} u_{j}\right)^{n}-\left(d d^{c} v_{j}\right)^{n}\right]=0$.

By using Theorem 3.1 we also obtain the following application.

Theorem 3.3. Let $u_{j}, u, v \in \mathscr{E}(\Omega)$ be such that $u_{j} \geq v, \forall j \geq 1$ and $\varlimsup_{j \rightarrow \infty} u_{j} \leq u$. Assume that the sequence of measures $\left(d d^{c} u_{j}\right)^{n}$ has a limit point $\mu$ in the weak-topology of measures. Then $\mu \geq 1_{\{u=-\infty\}}\left(d d^{c} u\right)^{n}$ in the weak sense of measures.

We need:

Lemma 3.4. Let $u \in \mathscr{E}(\Omega)$ and a compact subset $K$ in $\{u=-\infty\}$. Given open sets $D_{j} \subset \subset \Omega$ such that $\bar{D}_{j} \searrow K$. Then there exist $\varphi_{j} \in \mathscr{E}_{0} \cap C(\Omega)$ such that $\varphi_{j} \searrow u$ and $\lim _{j \rightarrow \infty} 1_{D_{j}}\left(d d^{c} \varphi_{j}\right)^{n}=1_{K}\left(d d^{c} u\right)^{n}$ in the weak-topology of measures.

Proof. By Theorem 2.1 in [7] we can find

$$
\mathscr{E}_{0} \cap C(\Omega) \ni \psi_{j} \searrow u .
$$

Since $\left(d d^{c} \psi_{j}\right)^{n} \rightarrow\left(d d^{c} u\right)^{n}$ as $j \rightarrow \infty$ we can find a increasing sequence $\left\{k_{j}\right\}$ such that

$$
\int_{D_{j}}\left(d d^{c} \psi_{k_{j}}\right)^{n} \geq \int_{D_{j}}\left(d d^{c} u\right)^{n}-\frac{1}{j} .
$$

Set $\varphi_{j}=\psi_{k_{j}}$. We have

$$
\lim _{j \rightarrow \infty} 1_{D_{j}}\left(d d^{c} \varphi_{j}\right)^{n} \leq \lim _{j \rightarrow \infty} 1_{\bar{D}_{k}}\left(d d^{c} \varphi_{j}\right)^{n}=1_{\bar{D}_{k}}\left(d d^{c} u\right)^{n}
$$

for all $k \geq 1$. Letting $k \rightarrow \infty$ we get

$$
\lim _{j \rightarrow \infty} 1_{D_{j}}\left(d d^{c} \varphi_{j}\right)^{n} \leq 1_{K}\left(d d^{c} u\right)^{n} .
$$


On the other hand, we have

$$
\begin{aligned}
\varliminf_{j \rightarrow \infty} \int_{\Omega} 1_{D_{j}}\left(d d^{c} \varphi_{j}\right)^{n} & =\varliminf_{j \rightarrow \infty} \int_{D_{j}}\left(d d^{c} \psi_{k_{j}}\right)^{n} \geq \underline{\lim }_{j \rightarrow \infty}\left[\int_{D_{j}}\left(d d^{c} u\right)^{n}-\frac{1}{j}\right] \\
& =\int_{\Omega} 1_{K}\left(d d^{c} u\right)^{n} .
\end{aligned}
$$

Therefore $\lim _{j \rightarrow \infty} 1_{D_{j}}\left(d d^{c} \varphi_{j}\right)^{n}=1_{K}\left(d d^{c} u\right)^{n}$.

Proof of Theorem 3.3. Let $K$ be a compact subset in $\{u=-\infty\}$. Given $f \in C_{0}^{\infty}(\Omega), f \geq 0$. We only have to prove that

$$
\int_{\Omega} f d \mu \geq \int_{\Omega} f 1_{K}\left(d d^{c} u\right)^{n}
$$

We choose open sets $D_{j} \subset \subset \Omega$ such that $D_{j} \searrow K$. By Lemma 3.3 we can find $\varphi_{j} \in \mathscr{E}_{0}(\Omega) \cap C(\Omega)$ such that $\varphi_{j} \searrow u$ and $\lim _{j \rightarrow \infty} 1_{D_{j}}\left(d d^{c} \varphi_{j}\right)^{n}=1_{K}\left(d d^{c} u\right)^{n}$. Since $\sup \left\{\varphi_{j}(z): z \in D_{j}\right\} \searrow-\infty$ we can assume that

$$
\sup \left\{\varphi_{j}(z): z \in D_{j}\right\}<-j(j+1)
$$

By [14] we can choose a increasing $\left\{k_{j}\right\}$ such that $u_{k_{j}} \leq \varphi_{j}+1$ on $\bar{D}_{j}$. Set

$$
v_{j}=\max \left(u_{k_{j}},\left(1-\frac{1}{j}\right) \varphi_{j}-j\right) .
$$

Since $u_{k_{j}} \leq \varphi_{j}+1<\left(1-\frac{1}{j}\right) \varphi_{j}-j$ on $D_{j}$ we get $v_{j}=\left(1-\frac{1}{j}\right) \varphi_{j}-j$ on $D_{j}$. This implies that

$$
\begin{aligned}
\lim _{j \rightarrow \infty} \int_{\Omega} f\left(d d^{c} v_{j}\right)^{n} & \geq \underset{j \rightarrow \infty}{\lim }\left(1-\frac{1}{j}\right)^{n} \int_{\Omega} f 1_{D_{j}}\left(d d^{c} \varphi_{j}\right)^{n} \\
& =\int_{\Omega} f 1_{K}\left(d d^{c} u\right)^{n}
\end{aligned}
$$

Since $\left\{\left|u_{k_{j}}-v_{j}\right| \neq 0\right\} \subset\left\{u_{k_{j}}<-j\right\} \subset\{v<-j\}$ we get $u_{k_{j}}-v_{j} \rightarrow 0$ in $C_{n}$-capacity. Moreover, by Theorem 3.1 we have

$$
\lim _{j \rightarrow \infty} \int_{\Omega} f\left(d d^{c} u_{k_{j}}\right)^{n}=\lim _{j \rightarrow \infty} \int_{\Omega} f\left(d d^{c} v_{j}\right)^{n} .
$$

From (1) and (2), letting $j \rightarrow \infty$ we get

$$
\int_{\Omega} f \mu \geq \int_{\Omega} f 1_{K}\left(d d^{c} u\right)^{n} .
$$




\section{REFERENCES}

1. Åhag, P., and Czyż, R., On the Cegrell classes, Math. Z. 256 (2007), 243-264.

2. Åhag, P., Cegrell, U., Czyż, R., and Hiep, P., Monge-Ampère on pluripolar sets, J. Math. Pures Appl. 92 (2009), 613-627.

3. Bedford, E., and Taylor, B. A., The Dirichlet problem for a complex Monge-Ampère equation, Invent. Math. 37 (1976), 1-44.

4. Bedford, E., and Taylor, B. A., A new capacity for plurisubharmonic functions, Acta Math. 149 (1982), 1-40.

5. Cegrell, U., Delta-plurisubharmonic functions, Math. Scand. 43 (1978), 343-352

6. Cegrell, U., Pluricomplex energy, Acta Math. 180 (1998), 187-217.

7. Cegrell, U., The general definition of the complex Monge-Ampère operator, Ann. Inst. Fourier (Grenoble) 54 (2004), 159-179.

8. Cegrell, U., Convergence in capacity, Newton Institute preprint NI01046-NPD (2001) (http://www.arxiv.org/).

9. Demailly, J.-P., Monge-Ampère operators, Lelong numbers and intersection theory, pp. 115193 in: Complex Analysis and Geometry, Univ. Ser. Math., Plenum, New York 1993.

10. Guedj, V., and Zeriahi, A., Intrinsic capacities on compact Kähler manifolds, J. Geom. Anal. 15 (2005), 607-639.

11. Guedj, V., and Zeriahi, A., The weighted Monge-Ampère energy of quasiplurisubharmonic functions, J. Funct. Anal. 250 (2007) 442-482.

12. Hiep, P. H., On the convergence in capacity on compact Kähler manifolds and its applications, Proc. Amer. Math. Soc. 136 (2008), 2007-2018.

13. Hiep, P. H., Convergence in capacity, Ann. Polon. Math. 93 (2008), 91-99.

14. Hörmander, L., Notion of Convexity, Progress in Math. 127, Birkhäuser, Boston 1994.

15. Khue, N. V., and Hiep, P. H., A comparison principle for the complex Monge-Ampère operator in Cegrell's classes and applications, Trans. Amer. Math. Soc. 361 (2009), 5539-5554.

16. Klimek, M., Pluripotential Theory, London Math. Soc. Monographs 6, Oxford Univ. Press, New York 1991.

17. Kołodziej, S., The range of the complex Monge-Ampère operator, II, Indiana Univ. Math. J. 44 (1995), 765'-782.

18. Kołodziej, S., The Monge-Ampère equation on compact Kähler manifolds, Indiana Univ. Math. J. 52 (2003), 667-686.

19. Xing, Y., Continuity of the complex Monge-Ampère operator, Proc. Amer. Math. Soc. 124 (1996), 457-467.

20. Xing, Y., Continuity of the complex Monge-Ampere operator on compact Kähler manifolds, Math. Z. 263 (2009), 331-344.

DEPARTMENT OF MATHEMATICS

HANOI UNIVERSITY OF EDUCATION (DAI HOC SU PHAM HA NOI)

CAU GIAY

HANOI

VIETNAM

E-mail: phhiep_vn@yahoo.com 